\title{
Study on Thermal Conductivities Prediction for Apple Fruit Juice by Using Neural Network
}

\author{
Min Zhang ${ }^{1, *}$, Zhenhua Che ${ }^{1}$, Jiahua $\mathrm{Lu}^{1}$, Huizhong Zhao ${ }^{2}$, Jianhua Chen ${ }^{1}$, \\ Zhiyou Zhong ${ }^{1}$, and Le Yang ${ }^{1}$ \\ ${ }^{1}$ College of Food Sciences, Shanghai Ocean University, Shanghai, 201306, P. R. China \\ zhangmashou.edu.cn \\ ${ }^{2}$ School of Environment and Architecture, University of Shanghai for Science and Technology, \\ Shanghai, 200093, P. R. China
}

\begin{abstract}
Based on experimentally measured values by thermal probe method, the prediction model of thermal conductivities of apple fruit juice as a function of concentration and temperature was studied by neural network method. The optimal neural network was made of two hidden layers and every hidden layer had six neurons. The prediction result shows that the optimal model could predict thermal conductivity with a mean relative error of $0.11 \%$, a mean absolute error of $0.00054 \mathrm{~W} / \mathrm{mK}$, a mean standard error of $0.00039 \mathrm{~W} / \mathrm{mK}$, the linear relationship of 0.9993 . The calculated precision was higher for BP neural network model than that for dual regression model. The presented results were proved that this model can be used with satisfactory accuracy for the prediction of thermal conductivity of apple fruit juice.
\end{abstract}

Keywords: Thermal conductivity, Neural network, Apple fruit juice.

\section{Introduction}

Apple fruit juice as a traditional consumption is widely available on the Chinese market. It is a complex mixture of vitamins, pectin, sugar, mineral salts, organic acids andamino acid. Accurate values of thermophysical parameters over a wide temperature range are needed in the fruit juice industry in order to design and optimize the operation of handling and processing units [1].

Thermal conductivity of fruit juice is one of the important thermophysical parameters used to estimate the rate of heat transfer during processes, such as preservation, transportation and freezing. Generally, mathematical models which express the dependence of thermophysical properties on temperature and concentration are a very appealing alternative to experimentation, and a useful tool for the implementation of computer-aided routines for equipment design and process automation [2].

Over the years an extensive investigation on existing methods of measurement of thermal conductivity of fruits juice has been carried out [1][2][3]. Some empirical equations have been proposed for the estimation of thermal conductivity of various

\footnotetext{
* Corresponding author.
} 
fruits juice products as a function of composition [4][5]. In these equations one can easily verify that thermal conductivity of fruits juice depends strongly on the water content, since water has the highest thermal conductivity of all food components [6].

In recent years, artificial neural networks as a kind of analysis method were obtained extensive attentions by food engineer. It has become increasingly popular during the last decade. The advantages of this method compared are its high speed, simplicity and large capacity which reduce engineering effort [7]. It can be used successfully in various fields of modeling and prediction in many engineering systems, mathematics, medicine, economics, meteorology and many others [8].

The objective of this study is to develop a BP artificial neural network model to predict the thermal conductivity of apple juice as a function of concentration and temperature and to compare the BP artificial neural network model established with dual regression model by the calculated precisions.

\section{Methodology}

The development of a neural network model involves: the generation of data required for training/testing, the training/testing of the BP artificial neural network model, the evaluation of the BP artificial neural network configuration leading to the selection of an optimal configuration, and validation of the optimal BP artificial neural network model with a data set not used in training before [9].

\subsection{Data Generation of Thermal Conductivity of Apple Juice}

The thermal conductivity of apple juice at different concentration and temperature were measured using a tiny thermal probe method. It has been described in detail in our previous studied [10]. In this study, the thermal conductivity probe system was proposed, which needs short duration of the experiments, simplicity, high accuracy and relatively small sample requirement. The system worked satisfactorily for its application. The effects of concentration in the range of $0-70^{\circ}$ Brix and temperature in the range of $3-50^{\circ} \mathrm{C}$ on the thermal conductivity of apple juice were determined. The 192 data sets with different thermal conductivities affected by concentrations and temperatures were obtained through using this measurement system. The thermal conductivity of apple juice decreased with an increase in concentration while it increased with an increase in temperature. In addition, the influence of concentration on the thermal conductivity of apple juice was stronger than that of temperature. A double-factors regressive equation of thermal conductivity of apple juice as a function of concentration and temperature was obtained:

$$
k=0.582+0.0012 t-0.0035 s
$$

Where $k$ is the thermal conductivity of apple juice, $\mathrm{W} / \mathrm{m} \mathrm{K} ; s$ is the concentration of the apple juice $\left({ }^{\circ}\right.$ Brix) and $t$ is the temperature of the apple juice, ${ }^{\circ} \mathrm{C}$.

\subsection{Establishment of BP Neural Network}

A multi-layer feed forward BP network structure with input, output and hidden layer(s) was used in this study as shown in Figure 1. It consists of the feed-forward 
pass and backward pass process. The input layer includes two neuron parameters of concentration and temperature. The output layer is the thermal conductivity of apple fruit juice, hidden layer suppose $n$ neurons before training.

The network is processed through two stages: training stage and validation stage [11]. BP neural network achieves full connectivity between the upper and lower layers, and no connections between neurons in each layer. The neural cells of input and output layers were decided by the practical input and output parameters [12]. In the training stage, the network was trained to predict an output based on input data. In the validation stage, the network was validated to stop or continue training. The neurons of hidden layer were decided on the error decline in the training course. The learning process was composed of positive signal transmission and the error back-propagation. The actual output value was calculated by the process information flow from input layer, hidden layer to output layer by layer. If the actual output of the output layer does not match with the expected output, the error back-propagation stage was started [13]. The error signals of neural cells were based on this error. The each unit weight can be amended by the error signal values until the network output error was reduced to tolerant ranges.

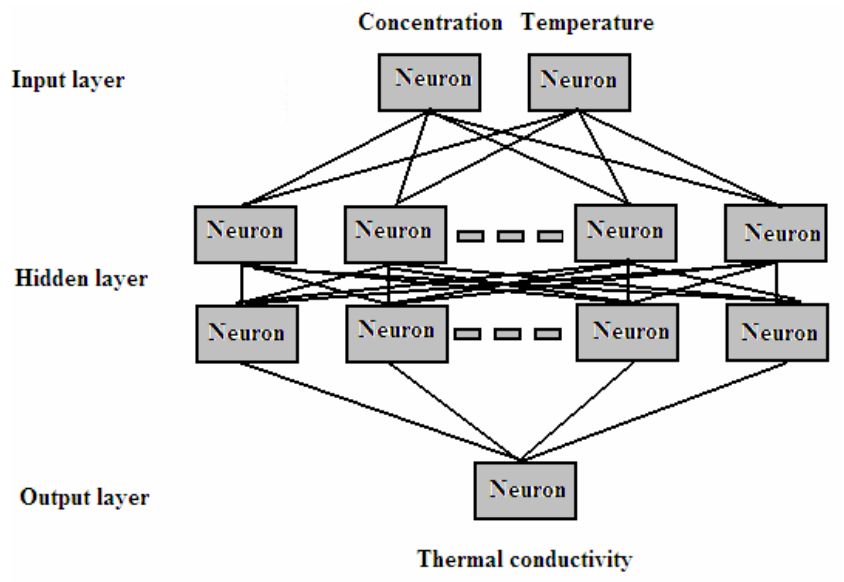

Fig. 1. Model of BP neural network

The indicators of thermal conductivity of apple fruit juice including the mean absolute error $M A E$, mean relative error $M R E$, standard deviation $S E$, coefficient of determination $R^{2}$ can judge the right type of performance of the network and optimize neural network model. The four indicators used to compare the performance of various BP artificial neural network configurations were calculated by the following expressions [9]:

$$
M A E=\frac{1}{N} \sum_{c=1}^{N}\left|k_{m}-k_{p}\right|
$$




$$
\begin{gathered}
M R E=\frac{1}{N} \sum_{c=1}^{N} \frac{\left|k_{m}-k_{p}\right|}{k_{m}} \\
S E=\sqrt{\frac{\sum_{c=1}^{N}\left(k_{m}-k_{p}\right)^{2}}{N-1}} \\
R^{2}=1-\sum_{c=1}^{N} \frac{\left(k_{m}-k_{p}\right)^{2}}{k_{m}^{2}}
\end{gathered}
$$

Where $N$ is the number of training samples; $k_{m}$ and $k_{p}$ are the actual measurement values and the neural network fitting calculated values of thermal conductivity.

\section{Results and Discussion}

The thermal conductivity data sets of the 144 cases in our study were divided into two groups; training and testing sets. The BP neural network model was trained using 116 randomly selected data (accounting for $80 \%$ of the total data) while the remaining 28 data (accounting for 20\%) was utilized for testing of the network performance. In order to improve training characteristics, the thermal conductivity data set experimentally obtained were normalized using a simple normalization method. The Maximum and minimum values of the inputs and outputs and normalization values ere given in Table 1.

Table 1. Input and output parameters ranges and normalization values

\begin{tabular}{cccc}
\hline Parameters & $\begin{array}{c}\text { Minimum } \\
\text { value }\end{array}$ & $\begin{array}{c}\text { Maximum } \\
\text { value }\end{array}$ & $\begin{array}{c}\text { Normalization } \\
\text { value }\end{array}$ \\
\hline Concentration, $\%$ & 0 & 70 & 70 \\
Temperature, ${ }^{\circ} \mathrm{C}$ & 3 & 50 & 40 \\
Thermal conductivity, $\mathrm{W} / \mathrm{mK}$ & 0.341 & 0.647 & 0.646 \\
\hline
\end{tabular}

The modeling program was implemented under MATLAB. The BP learning algorithm has been used in feed-forward, which included an input layer, a hidden layer and an output layer network. There are two input parameters in the input layer, namely the temperature difference and concentration of the apple juice. The output is the thermal conductivity of apple juice. The number of neurons in the input layer is equal to the number of input parameters and the number of neurons in the output layer is the number of output parameters. Optimal number of the neurons in the hidden layer was determined by trying different networks[8]. The number of neurons was increased from 1 to 8 based on the trial and error method in the hidden layer. 
Input layer and hidden layer use the sigmoid transfer function for the transfer function, the output layer use the purelin function, in order to improve the prediction accuracy, the test results would be normalized, so that concentration $C_{i} \in[0,1]$, temperature $t_{i} \in[0,1]$, thermal conductivity $k_{m} \mathrm{i} \in[0,1]$. In the process of model, the network is initialized by means of Newff function in Matlab neural network toolbox, the trained model parameters set as follows: the maximum epoch of 10000 , network performance goal error of $10^{-6}$, learning rate of 0.02 , momentum constant of 0.9 . The errors associated with desired output response are adjusted in the way that reduces these errors in each neuron from the output to the input layer. When the network output error was reduced to the required tolerance, the training automatically stopped. According to equation (2) - (5) the neural network established was training and the error of the fitting calculation results of each network was analyzed. It is found that best structure of the model has six neurons in the first and second hidden layer presented in Table 2. The MRE, MAE, SE and $R^{2}$ for this optimal configuration were $0.92 \%, 0.0047 \mathrm{~W} / \mathrm{mK}, 0.000525 \mathrm{~W} / \mathrm{mK}$, and 0.9957 , respectively. So the optimized BP neural network model architecture has a configuration of 2-4-4-1 neurons.

Table 2. Error parameters with different BP neural network structure

\begin{tabular}{cccccc}
\hline $\begin{array}{c}\text { Hidden } \\
\text { layers }\end{array}$ & Neurons & $\begin{array}{c}\text { MRE } \\
(\%)\end{array}$ & $\begin{array}{c}M A E \\
(\mathrm{~W} / \mathrm{m} \cdot \mathrm{K})\end{array}$ & $\begin{array}{c}S E \\
(\mathrm{~W} / \mathrm{m} \cdot \mathrm{K})\end{array}$ & $R^{2}$ \\
\hline 1 & 1 & 1.11 & 0.0056 & $6.66 \mathrm{E}-04$ & 0.9930 \\
1 & 2 & 1.04 & 0.0052 & $6.13 \mathrm{E}-04$ & 0.9941 \\
1 & 4 & 1.09 & 0.0055 & $6.49 \mathrm{E}-04$ & 0.9933 \\
2 & 2 & 1.07 & 0.0054 & $6.25 \mathrm{E}-04$ & 0.9938 \\
$\mathbf{2}$ & $\mathbf{4}$ & $\mathbf{0 . 9 2}$ & $\mathbf{0 . 0 0 4 7}$ & $\mathbf{5 . 2 5 E}-\mathbf{0 4}$ & $\mathbf{0 . 9 9 5 7}$ \\
2 & 6 & 0.97 & 0.0049 & $5.65 \mathrm{E}-04$ & 0.9950 \\
\hline
\end{tabular}

The prediction thermal conductivity of apple juice for the optimized BP neural network model with a configuration of 2-4-4-1 neurons were compared with the experimental ones as shown in Figure 2 for the 116 training data set. The results demonstrated good agreement between the predicted and the experimental values of thermal conductivity $\left(R^{2}=0.9957\right)$.

The performance of the optimal neural network (two hidden layers and six neurons in each hidden layer) was validated using a smaller data set consisting 28 cases. Correlation between predicted and measured values of thermal conductivity was shown in Figure 3.This network predicted thermal conductivity values with an MRE of $0.11 \%$, $M A E$ of $0.00054 \mathrm{~W} / \mathrm{mK}$ and $S E$ of $0.00039 \mathrm{~W} / \mathrm{mK}$, respectively. It still kept a good agreement between the predicted and the measured values of thermal conductivity $\left(R^{2}=0.9993\right)$. The results presented that this model can be used with satisfactory accuracy for the prediction of thermal conductivity of apple fruit juice. The mean relative error of 28 prediction values of thermal conductivity cases in this model is smaller than that in double-factors regressive equation whose mean relative error is $1.73 \%$ [10]. 


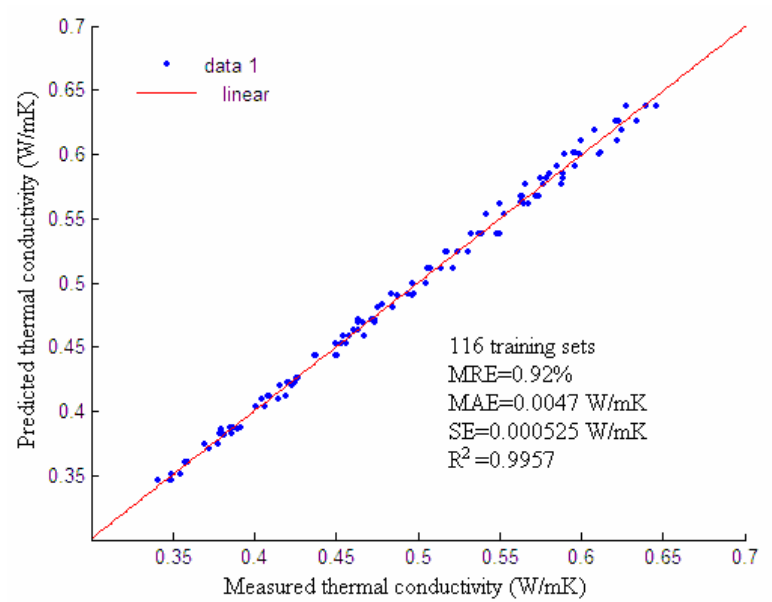

Fig. 2. Comparison of measured and predicted values of thermal conductivity for the training sets

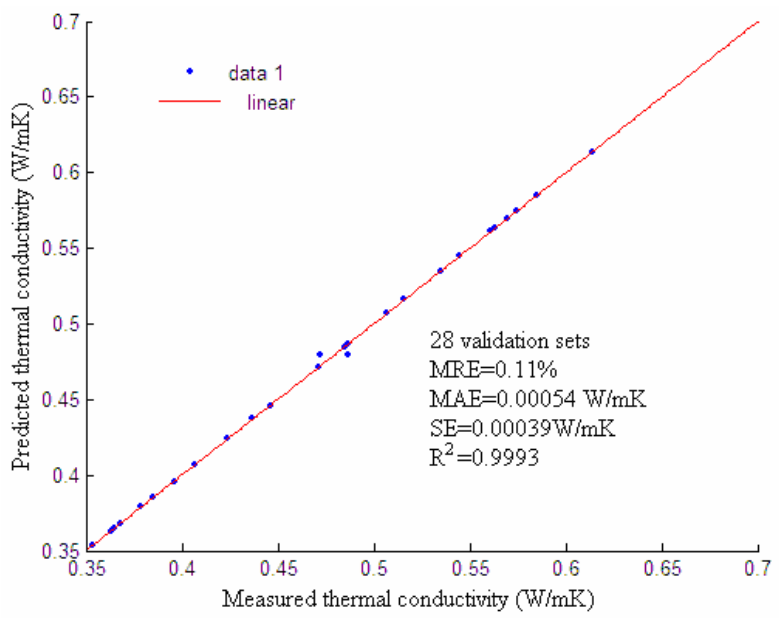

Fig. 3. Comparison of measured and predicted values of thermal conductivity for the validation sets

\section{Conclusion}

In this work, the prediction model of thermal conductivities of apple fruit juice as a function of concentration and temperature was studied by neural network method. The optimal neural network was made of two hidden layers and every hidden layer had six neurons. The optimal neural model could predict thermal conductivity with $M R E$ of $0.11 \%, M A E$ of $0.00054 \mathrm{~W} / \mathrm{mK}, S E$ of $0.00039 \mathrm{~W} / \mathrm{mK}, R^{2}$ of 0.9993 while the mean relative error in double-factors regressive equation was $1.73 \%$. The prediction 
precision of BP neural network model was higher than that of dual regression model. The presented results were proved that this neural network model can be used with satisfactory accuracy for the prediction of thermal conductivity of apple fruits juice and further applications.

Acknowledgments. Funding for this research was provided by the Natural Science Foundation of China, Grant No 30771245 and Leading Academic Discipline Project of Shanghai Municipal Education Commission, Project No J50704.

\section{References}

1. Frandas, A., Bicanic, D.: Thermal properties of fruit juices as a function of concentration and temperature determined using the photopyroelectric (PPE) method. Journal of the Science of Food and Agriculture 79, 1361-1366 (1999)

2. Telis-Romero, J., Telis, V.R.N., Gabas, A.L., Yamashitah, F.: Thermophysical Properties of Brazilian Orange Juice as Affected by Temperature and Water Content. Journal of Food Engineering 38, 27-40 (1998)

3. Shamsudin, R., Mohamed, I.O., Yaman, N.K.M.: Thermophysical properties of Thai seedless guava juice as affected by temperature and concentration. Journal of Food Engineering 66, 395-399 (2005)

4. Zainal, B.S., Abdul Rahman, R., Ariff, A.B., Saari, B.N., Asbi, B.A.: Effects of temperature on the physical properties of pink guava juice at two different concentrations. Journal of Food Engineering 43, 55-59 (2000)

5. Azoube, P.M., Cipriani, D.C., El-Aouar, A.A., Antonio, G.C., Murr, F.E.X.: Effect of concentration on the physical properties of cashew juice. Journal of Food Engineering 66, 413-417, 79, 1361-1366 (1999)

6. Saravacos, G.D., Kostaropoulos, A.E.: Transport properties in processing of fruits and vegetables. Food Technol 49, 99-109 (1995)

7. Kalogirou, S.A.: Artificial neural networks in the renewable energy systems applications: a review. Renew Sustain Energy Rev. 5, 373-401 (2001)

8. Kurt, H., Kayfeci, M.: Prediction of thermal conductivity of ethylene glycol-water solutions by using artificial neural networks. Applied Energy 86, 2244-2248 (2009)

9. Sablani, S.S., Baik, O.-D., Marcotte, M.: Neural networks for predicting thermal conductivity of bakery products. Journal of Food Engineering 52, 299-304 (2002)

10. Zhang, M., Zhao, H.Z., Xie, J., Sun, Z.Q., Zhang, B.L.: Experimental study on influence factors of thermal conductivities of apple juice. Transactions of the CSAE 22, 162-165 (2006)

11. Yuan, Q.X.: Prediction for the Effect of Temperature and Water Content on the Soil Specific Heat by BP Neural Network. Transactions of the CSAE 39, 108-111 (2008)

12. Zhang, M., Zhong, Z.Y., Yang, L., Zhao, H.Z., Chen, J.H., Che, Z.H.: Prediction model of thermal conductivities of fruits and vegetables based on BP neural networks. Transactions of the CSAE 41, 117-121 (2010)

13. Xu, X., Yu, Z.T., Hu, Y.C., Fan, L.W., Tian, T., Cen, K.F.: Nonlinear fitting calculation of wood thermal conductivity using neural networks. Journal of Zhejiang University 41, 1201-1204 (2007) 